Draft VERSION June 3, 2018

Preprint typeset using $\mathrm{LAT}_{\mathrm{E} X} \mathrm{X}$ style emulateapj v. 02/07/07

\title{
THE LUMINOSITY FUNCTION OF THE MILKY WAY SATELLITES
}

\author{
S. Koposov ${ }^{1,2}$, V. Belokurov ${ }^{2}$, N.W. Evans ${ }^{2}$, P.C. Hewett ${ }^{2}$, M.J. Irwin ${ }^{2}$, G. Gilmore ${ }^{2}$, D.B. Zucker ${ }^{2}$, H.-W. Rix $^{1}$, \\ M. Fellhauer ${ }^{2}$, E.F. Bell ${ }^{1}$, E.V. Glushkova ${ }^{3}$ \\ Draft version June 3, 2018
}

\begin{abstract}
We quantify the detectability of stellar Milky Way satellites in the Sloan Digital Sky Survey (SDSS) Data Release 5 . We show that the effective search volumes for the recently discovered SDSS-satellites depend strongly on their luminosity, with their maximum distance, $D_{\max }$, substantially smaller than the Milky Way halo's virial radius. Calculating the maximum accessible volume, $V_{\max }$, for all faint detected satellites, allows the calculation of the luminosity function for Milky Way satellite galaxies, accounting quantitatively for their detectability. We find that the number density of satellite galaxies continues to rise towards low luminosities, but may flatten at $M_{V} \sim-5$; within the uncertainties, the luminosity function can be described by a single power law $d N / d M_{V}=10 \times 10^{0.1\left(M_{V}+5\right)}$, spanning luminosities from $M_{V}=-2$ all the way to the luminosity of the Large Magellanic Cloud. Comparing these results to several semi-analytic galaxy formation models, we find that their predictions differ significantly from the data: either the shape of the luminosity function, or the surface brightness distributions of the models, do not match.
\end{abstract}

Subject headings: Galaxy: halo - Galaxy: structure - Galaxy: formation - Local Group

\section{INTRODUCTION}

In Cold Dark Matter (CDM) models, large spiral galaxies like the Milky Way and M31 form within extensive dark matter halos from the merging and accretion of smaller systems. Although CDM models have had many successes on larger scales, one of the most serious challenges facing CDM models is the so-called "miss-

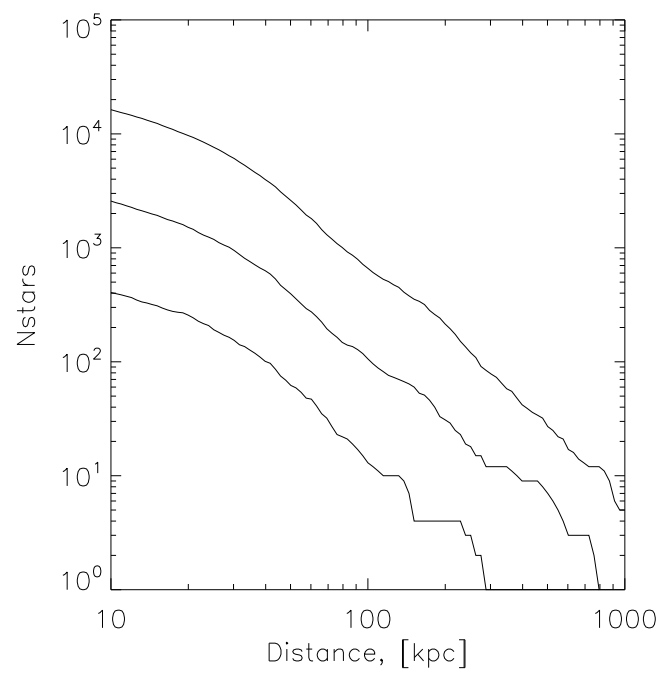

FIG. 1.- The number of stars brighter than $r \simeq 22.5$ in random realizations of Milky Way satellites of luminosity $M_{r} \sim-3,-5,-7$ (from bottom to top) with M92-like stellar populations, as a function of distance from the Sun. The approximate number of stars required for a significant detection (by the algorithm described in Section 2) is $\simeq 30$.

\footnotetext{
${ }^{1}$ Max Planck Institute for Astronomy, Königstuhl 17, 69117 Heidelberg, Germany

${ }^{2}$ Institute of Astronomy, Madingley Road, Cambridge CB3 0HA, UK;koposov, vasily, nwe@ast.cam.ac.uk

3 Sternberg Astronomical Institute, Universitetskiy pr., 13, 119992, Moscow, Russia
}

ing satellite" problem. First identified by Klypin et al. (1999) and Moore et al. (1999), the problem manifests itself through the prediction by CDM models of at least 1-2 orders of magnitude more low-mass sub-halos at the present epoch compared to the observed abundance of dwarf galaxies surrounding the Milky Way and M31.

There have been a number of theoretical proposals to solve this problem. For example, the satellites that are observed could be embedded only in the rarer, more massive dark sub-halos (Stoehr et al. 2002), or, the satellites may form only in the rare peaks of halos that were above a given mass at reionization (Diemand et al. 2005; Moore et al. 2006). Alternatively, star formation in low mass systems could be inhibited by photoionization in the early Universe (Bullock et al. 2001; Somerville 2002; Benson et al. 2002). All these ideas do not alter the abundance of dark matter sub-halos, but propose to solve the observed discrepancy by producing a smaller number of directly observable satellites, thus breaking any simple relationship between mass and luminosity.

The known Milky Way dwarf spheroidal (dSph) satellites have been discovered by a variety of methods. The first seven were discovered serendipitously by visual inspection of photographic plates, the Sextans dSph was found using automated scans of photographic plates and the Sagittarius dSph in a radial velocity survey of the Milky Way bulge. All-sky photographic surveys cover most of the sky away from the Zone of Avoidance, but searches of plates are limited to surface brightnesses of $\sim 25.5 \mathrm{mag} \operatorname{arcsec}^{-2}$ (Whiting et al. 2007). The sample of known dSphs has long been bedeviled with selection effects, which are difficult to model with any accuracy. This situation has changed recently with the advent of very large area, homogeneous, photometric surveys such as the Sloan Digital Sky Survey (SDSS; York et al. 2000). The SDSS makes it possible to carry out a systematic survey for satellite galaxies, which are detectable through their resolved stellar populations down to extremely low surface brightnesses. In essence, SDSS greatly facilitates 


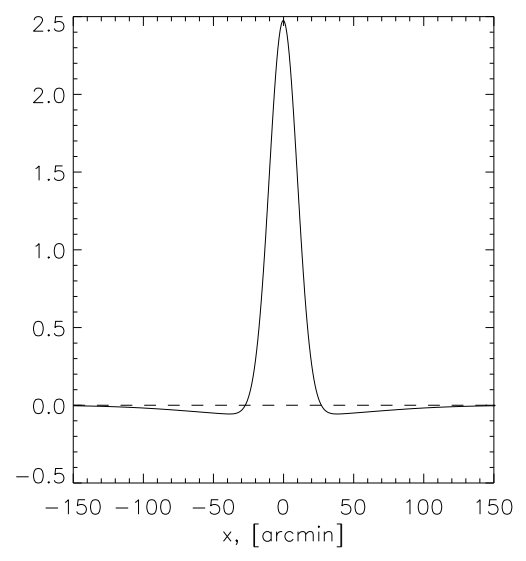

FIG. 2.- Differential convolution kernel applied to the stellar catalog to identify overdensities of a particular scale. A onedimensional slice of the two-dimensional kernel is shown, where the width, or $\sigma$, of the inner Gaussian is $6^{\prime}$ and of the outer Gaussian is $60^{\prime}$.

systematic searches for overdensities of stars in positioncolor-magnitude space.

Willman et al. (2002) carried out the first SDSSbased survey for resolved Milky Way satellites, subsequently discovering a new dwarf galaxy, Ursa Major (Willman et al. 2005a) as well as an unusually large globular cluster, Willman 1 (Willman et al. 2005b) - although later evidence may favor its interpretation as a dark matter dominated dwarf galaxy with multiple stellar populations (Martin et al. 2007). The color image "Field of Streams" (Belokurov et al. 2006a), composed of magnitude slices of the stellar density in the SDSS around the North Galactic Cap, proved to be a treasuretrove for dwarf galaxies, as Canes Venatici, Bootes I and Ursa Major II (Zucker et al. 2006a, b; Belokurov et al. $2006 \mathrm{~b}$ ) were all found in quick succession. A systematic search in the "Field of Streams" led to the discovery of five more satellite galaxies, Canes Venatici II, Leo IV, Hercules, Coma, and Leo T, as well as another large globular cluster, Segue 1 (Belokurov et al. 2007; Irwin et al. 2007). Very recently, Walsh et al. (2007) discovered another low luminosity satellite, Bootes II.

As the faintest Milky Way satellites currently constitute our best markers of sub-halos, the faint end of the satellite luminosity function of the Milky Way satellites can provide stringent constraints on the process of galaxy formation, and can distinguish between a number of dark matter, structure formation and reionization models. So, it is important not merely to carry out a systematic survey of the star overdensities in SDSS data for the discoveries per se, but also to compute the detection limits. These detection limits are the basis for a volume corrected luminosity function estimate and ultimately for a quantitative connection of satellite frequency and subhalo abundance. Such is the purpose of this paper. It is important to note that for a volume-corrected estimate of the luminosity function, it is not necessary to use exactly the same detection algorithms as Belokurov et al. (2007) or Willman et al. (2005a). Similarly, the detection scheme does not need to be optimal for every individual dwarf galaxy. Provided the automated algorithm is able to detect all the Milky Way satellites, and the completeness properties of the algorithm are quantified, an estimate of the true luminosity function can be derived.

\section{DETECTION OF SATELLITE GALAXY CANDIDATES IN SDSS DR5}

The SDSS Data Release 5 (DR5) covers $\sim 1 / 5$ of the sky, or $\sim 8000$ square degrees around the North Galactic Pole. SDSS imaging data are produced in five photometric bands, $u, g, r, i$, and $z$ (Fukugita et al. 1996; Gunn et al. 1998; Hogg et al. 2001; Adelman-McCarthy et al. 2006; Gunn et al. 2006). The data are automatically processed through pipelines to measure photometric and astrometric properties (Lupton, Gunn, \& Szalav 1999; Stoughton et al. 2002; Pier et al. 2003; Tucker et al. 2006). All magnitudes quoted in this paper have been corrected for reddening due to Galactic extinction using the maps of Schlegel, Finkbeiner, \& Davis (1998). Sometimes it is convenient to report our results in the $V$ band, for which we use the transformation $V=g-0.55(g-r)-0.03$ given by Smith et al. (2002).

The SDSS data with the source catalogs used in this paper was downloaded from the SAI CAS Virtual Observatory data center ${ }^{4}$ Koposov et al. 2007) and was stored locally in the PostgreSQL database. To perform queries rapidly on the large dataset, we used the Q3C plugin for the spatial queries (Koposov \& Bartunov 2006).

All the recent SDSS discoveries of $\mathrm{dSph}$ around the Milky Way, bar Leo T, are not directly visible in the fluxlimited images, but were detected as overdensities of resolved stars within certain magnitude and color ranges. This makes it straightforward to automate a detection method and assess its efficiency. The essence of any detection algorithm is to count the number of stars in a certain (angular) region on the sky, satisfying specified color and magnitude criteria, and compare the number to the background value. The excess of stars depends on the satellite's luminosity and distance. For a given luminosity, the distance fixes the number of stars brighter than the SDSS limiting magnitude, which is given by an integral over the stellar luminosity function. A simple illustration of the detectability of objects with a luminosity function like that of M92 is shown in Figure 1. The curves show the number of stars brighter than $r=22.5$ for satellites of three different absolute magnitudes. The maximal distance probed by surveys like SDSS is controlled by the apparent magnitude of the brightest stars in the satellite. For intrinsically luminous objects, like CVn I $\left(M_{V}=-7.9\right)$, we can detect stars at the tip of the red giant branch at distances of up to $\sim 1 \mathrm{Mpc}$. However, for satellites with many fewer stars, like Hercules $\left(M_{V}=-5.7\right)$, the giant branch tip is simply not populated and we can only detect objects at distances up to $\sim 300 \mathrm{kpc}$.

To identify the excess number of stars associated with a satellite, a common approach is to convolve the spatial distribution of the data with window functions or filters ${ }^{5}$. To estimate the star density on different scales,

\footnotetext{
4 http://vo.astronet.ru

5 This idea has a long history, particularly in algorithms for searching for features and clusters in imaging data. Widely used in
} 
we use a Gaussian of width $\sigma$, that is,

$$
L(x, y, \sigma)=I(x, y) * g(x, y, \sigma),
$$

where

$$
g(x, y, \sigma)=\frac{1}{2 \pi \sigma^{2}} \exp \left(-\frac{x^{2}+y^{2}}{2 \sigma^{2}}\right)
$$

and $I(x, y)$ is the distribution of sources

$$
I(x, y)=\sum_{i} \delta\left(x-x_{i}, y-y_{i}\right)
$$

This allows us to see the stellar density distribution at different spatial scales. For example, structures with a characteristic size of $1^{\prime}$ will be more prominent when the stellar map is convolved with a $1^{\prime}$ kernel, and less prominent when the map is convolved with $10^{\prime}$ and $0.1^{\prime}$ kernels. The resulting "blobs", or overdensities, can be easily identified on the differential image maps, namely

$$
\begin{aligned}
\Delta L & =L\left(x, y, \sigma_{1}\right)-L\left(x, y, \sigma_{2}\right) \\
& =I(x, y) *\left(g\left(x, y, \sigma_{1}\right)-g\left(x, y, \sigma_{2}\right)\right)
\end{aligned}
$$

Such differential image maps are generally convolutions of the original distribution with the kernel, which is a difference of two Gaussians. A one-dimensional slice of a kernel is shown in Figure 2, When we convolve the map $I(x, y)$ with such a kernel, we obtain an estimate of the local density minus an estimate of the local background $\left(L\left(x, y, \sigma_{2}\right)\right)$. This interpretation allows us to quantify the significance as

$$
S\left(x, y, \sigma_{1}, \sigma_{2}\right)=\frac{\Delta L}{\sigma_{L}}
$$

where $\sigma_{L}^{2}$ is the variance of $L\left(x, y, \sigma_{1}\right)$.

$$
\begin{aligned}
\sigma_{L}^{2} & =\operatorname{Variance}\left(L\left(x, y, \sigma_{1}\right)\right)= \\
& =\operatorname{Variance}\left(I(x, y) * g\left(x, y, \sigma_{1}\right)\right)=I(x, y) * g^{2}\left(x, y, \sigma_{1}\right)= \\
& =\sum_{i, j} I\left(x_{i}, y_{j}\right) g^{2}\left(x-x_{i}, y-y_{j}, \sigma_{1}\right) \approx \\
& \approx \sum_{i, j} L\left(x, y, \sigma_{2}\right) g^{2}\left(x-x_{i}, y-y_{j}, \sigma_{1}\right)= \\
& =L\left(x, y, \sigma_{2}\right) \iint g^{2}\left(x, y, \sigma_{1}\right) d x d y=\frac{L\left(x, y, \sigma_{2}\right)}{4 \pi \sigma_{1}^{2}}
\end{aligned}
$$$$
S\left(x, y, \sigma_{1}, \sigma_{2}\right)=\sqrt{4 \pi} \sigma_{1} \frac{\Delta L}{\sqrt{L\left(x, y, \sigma_{2}\right)}},
$$

Under the assumption that $\sigma_{2}>\sigma_{1}$ and a Poisson distribution of the initial set of datapoints, the variance of $S(x, y)$ is unity. This fact allows us to use the map of $S(x, y)$ to identify overdensities above a specified significance threshold.

\section{APPLICATION TO SDSS DATA}

SDSS's morphological parameters (Lupton et al. 2001) derived from the imaging data allow robust discrimination between stars and galaxies down to $r=21.5$. For

astronomy are kernel-based density estimation methods, in which the density is obtained by convolving all the data points (interpreted as delta-functions) with smoothly decaying kernels, which can be Gaussians (see e.g. Silverman 1986). A variant of this is used for feature detection in digital images in so-called scale-space science (Lindenberg 1993, 1998; Babaud et al. 1986).

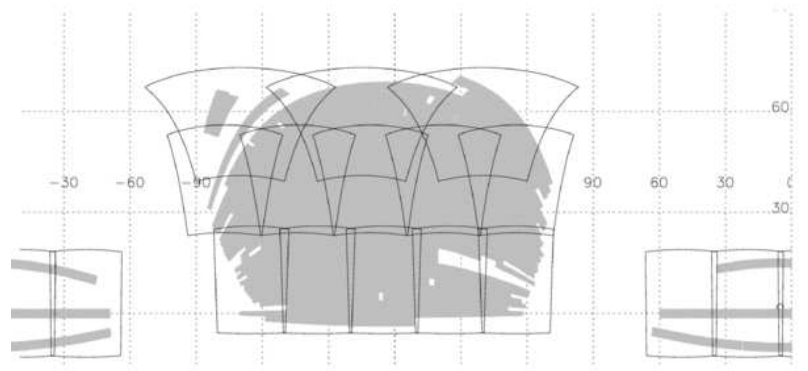

FIG. 3.- The segmentation of the DR5 area into $1732^{\circ} \times 32^{\circ}$ fields, used for the stellar overdensity search described in the text.

$21.5<r \lesssim 22.5$, the discrimination is still reasonably reliable, but it becomes increasingly untrustworthy below $r=22.5$. Moreover, the catalog is $95 \%$ complete at $r=22.2$ (Stoughton et al. 2002) and drops quickly below this magnitude. At the faint end, the "stellar" catalog of unresolved sources is polluted by faint galaxies which are intrinsically strongly clustered. We will see shortly that the main task in providing a clean sample of dwarf galaxy candidates is removal of the extragalactic contaminants, for which we will employ the SDSS galaxy catalog.

To proceed with the convolution (Eq. 4), the DR5 field of view is split into 17 segments as shown in Figure 3. The division is for computational convenience and to minimize distortion in the gnomonic projections. In practice, we select stars and galaxies with a magnitude cut-off of $r<22.5$. Due to the properties of the kernel, we expect edge effects at the boundaries of the DR5 footprint and we discard all overdensities within $1^{\circ}$ of a boundary. We use a color-cut of $g-r<1.2$ and kernel sizes with $\sigma_{1}=4^{\prime}$ and $\sigma_{2}=60^{\prime}$. The color cut is chosen to be as conservative as possible as regards inclusion of the tip of the red giant branch stars for metal-poor populations, whilst the kernel size is of the order of the angular size of the known dwarfs (see next section for details). The color magnitude cut used in this work may not be optimal for the detection of each individual dwarf galaxy (e.g. the isochrone masks should definitely work better), but the primary goal here is not to define an optimal (6) algorithm, but rather to develop a consistent algorithm that can detect known objects, for which the detection efficiency can be determined. Figure 4 provides an example of the application of the detection pipeline to the stellar and galaxy catalogs of SDSS DR5. The method successfully removes the varying background to leave underdensities (black regions) and overdensities (white regions). The sample field of view chosen for Figure 4 contains the already known Milky Way satellites Willman 1 and Ursa Major I (Willman et al. 2005a,b). They are both recovered in the stellar map with significances of $S_{\text {star }}=13.07$ and 5.95 respectively. However, as we see in Figure 4, unresolved sources in rich galaxy clusters such as Abell 773 and 1000, visible as prominent overdensities in the galaxy map, also show up in the stellar map as significant peaks.

In order to remove false positives caused by galaxy clustering, we need to understand the significance $S_{\text {gal }}$ of overdensities in the map derived from the galaxy catalog. Equation (7) does not hold, because the underlying distribution is no longer Poissonian (Figure 5). The left 


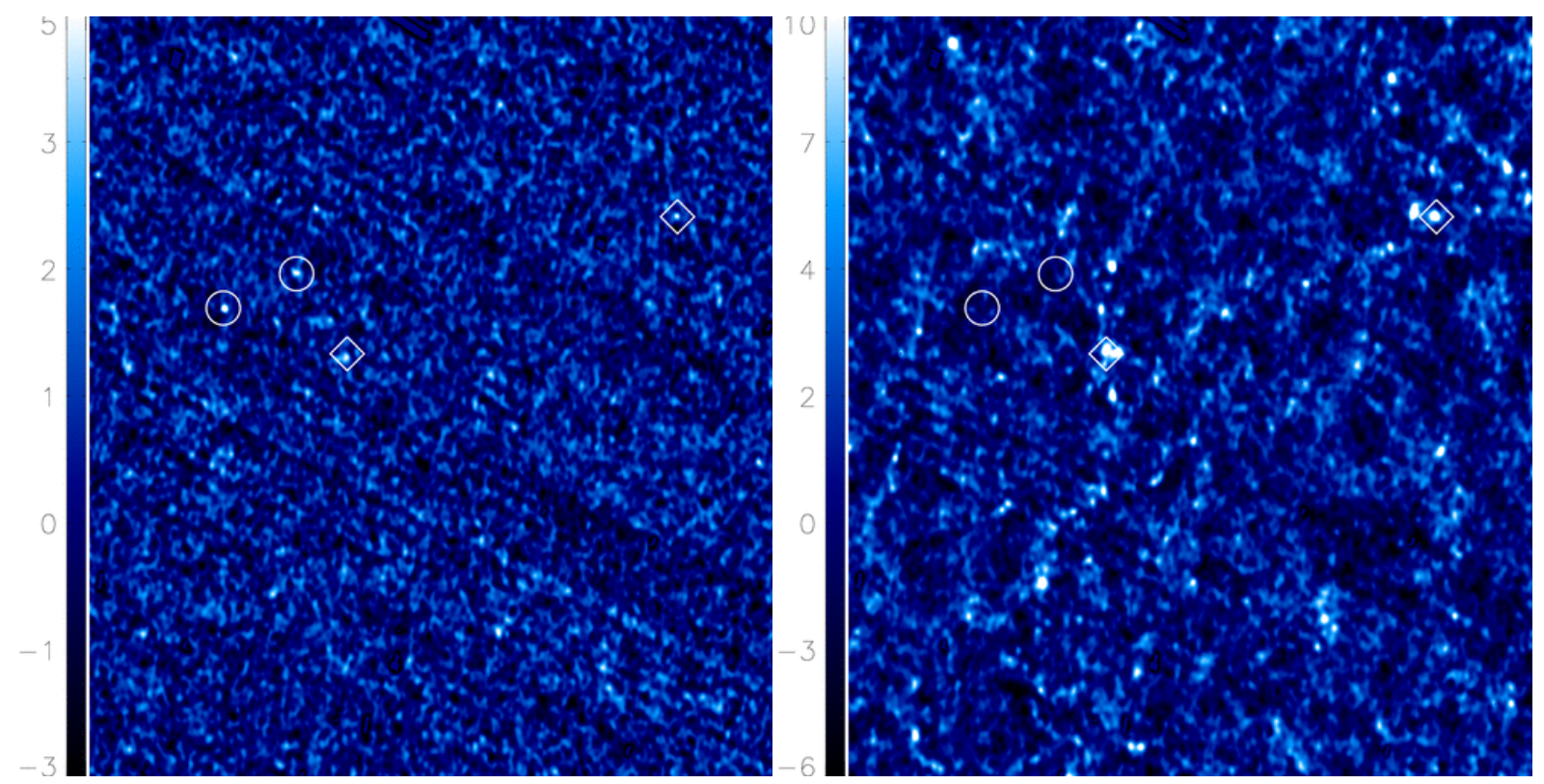

FIG. 4.- A sample $22^{\circ} \times 22^{\circ}$ area in the convolved maps of the SDSS DR5 stellar (left) and galaxy (right) catalogs. The positions of objects Ursa Major I and Willman 1 are marked by circles. The positions of galaxy clusters Abell 773 and Abell 1000 are marked by diamonds, and demonstrate that galaxy clusters may lead to significant peaks in the stellar map. The linear diagonal structures seen in both images are caused by SDSS stripes. The images were produced using a kernel specified by $\sigma_{1}=4^{\prime}$ and $\sigma_{2}=60^{\prime}$. We reject peaks in the convolved stellar map if they coincide with significant peaks in the galaxy distribution.
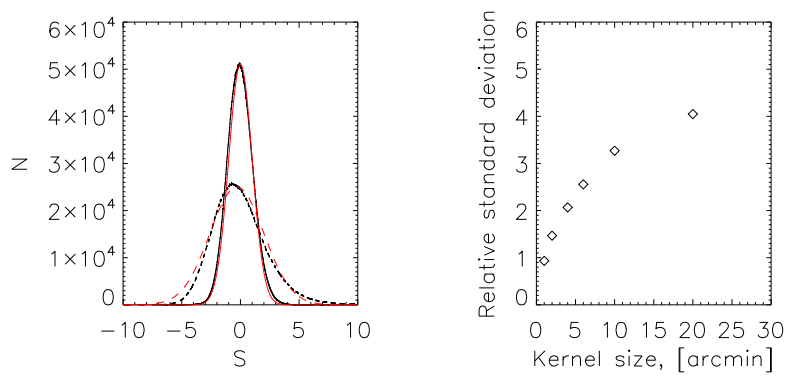

FIG. 5.- Left: The distribution of pixel values in the convolved star map (solid line) and galaxy map (dashed line) for one of our 17 fields in DR5. The Gaussian model curves with width of 1.0 and 2.3 centered on zero are shown in red. Right: The standard deviation in the galaxy map normalized by a Poissonian standard deviation as a function of kernel size.

panel shows the distributions of $S_{\text {star }}$ and $S_{\text {gal }}$ for all pixels in the same field of view as Figure 4. For the stars, the convolved source count distribution is almost a Gaussian with unit standard deviation, whilst the distribution for the galaxies is broader. The right panel shows how the width of the $S_{\text {gal }}$ distribution grows with increasing kernel width as the convolution samples coherent structures on larger scales. To assign significance to the overdensities in galaxies, we rescale $S_{\text {gal }}$, dividing by its standard deviation.

Next, we remove obvious false positives by rejecting all objects within the region marked by dashed lines in Figure 6. namely the intersection of the regions $S_{\text {star }}<20$ and $S_{\text {gal }}>2$. This removes most, but not all, the false positives caused by galaxy clusters. Additionally, there remains contamination from galaxies with large angular size. The SDSS photometric pipeline mis-classifies HII regions and stellar clusters in these galaxies as stars. We remove the contaminants by cross-correlating with the positions of galaxies in the Third Reference Cata-

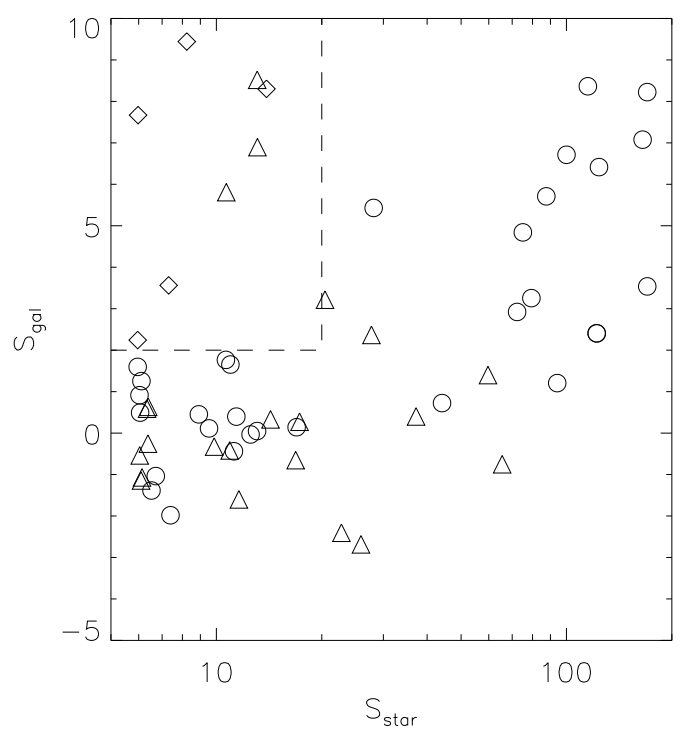

FIG. 6.- Distribution of Milky Way satellite detections in the $S_{\text {star }}$ versus $S_{\text {gal }}$ plane. The circles mark the known Milky Way satellites, the triangles are RC3 galaxies, and the rhomboids are galaxy clusters. Objects towards the top left of the figure are likely the result of contamination by galaxy clusters or spatially extended galaxies. The decision boundary is shown as a dashed line; objects to the right and below the dashed line are selected as candidate satellites.

logue (RC3) of de Vaucouleurs et al. (1991). Even so, there still remain objects at a moderately high level of significance whose nature is unclear. Most of these are probably caused by galaxy clusters or photometry artifacts, as judged from examination of Hess diagrams and SDSS image cut-outs, but there may still be a very small number of genuine Milky Way satellites.

We detect all the known Milky Way satellites, except Boo II, in a catalog with magnitude limit $r<22.5$, an- 

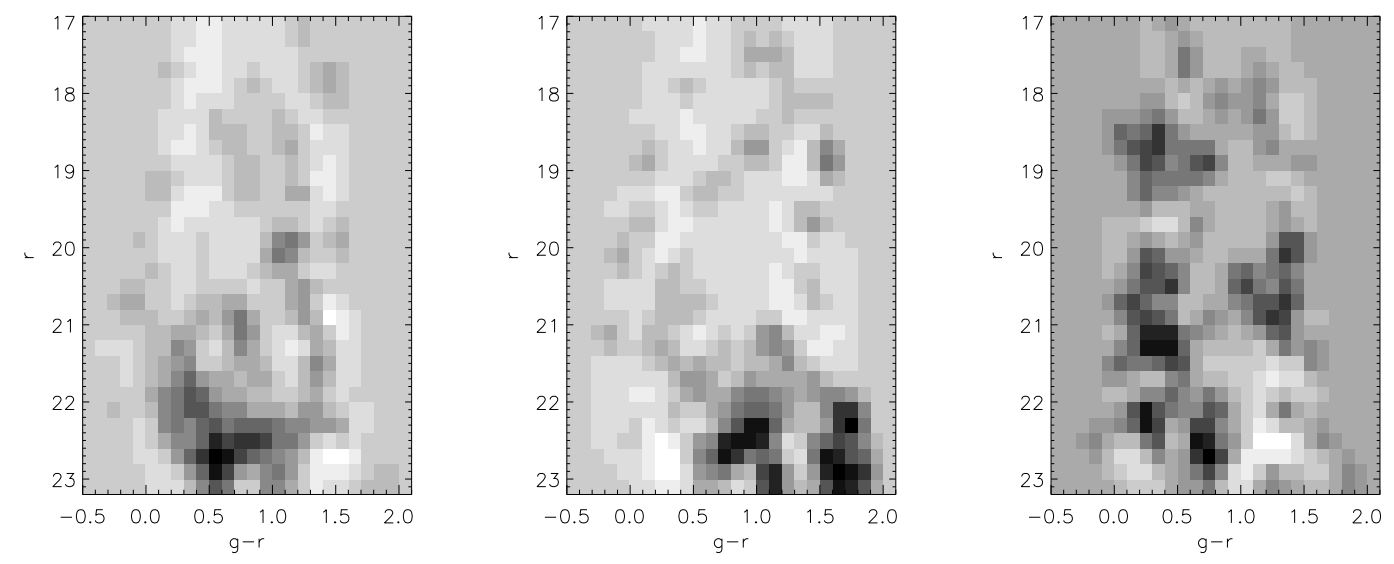

FIG. 7.- Left to right: Hess diagrams for Candidates X, Y and Z listed in Table 1

alyzed using a kernel with $\sigma_{1}=4^{\prime}$. The most marginal detections are Leo IV and Ursa Major I, which have significances $S_{\text {star }}=6.10$ and 5.95 respectively. Objects above the threshold are listed in Table 1, and include three likely false positives, which are "Candidates" X, $\mathrm{Y}$ and $\mathrm{Z}$. The Hess diagrams of these three detections are shown in Figure 7 . The Hess diagrams offer little evidence to support identification of the candidates as genuine satellites. Deeper data are needed to provide definitive classification of the candidates but for the purpose of determining the satellite luminosity function we exclude all three candidates, as false positives, from further consideration.

It is prudent to search for candidate satellites on the map convolved with different inner kernels, since the kernel biases the algorithm towards objects of a preferred size. Therefore, we performed a search on the map convolved with kernels of $2^{\prime}$ and $8^{\prime}$. In the former case, setting the significance to $S_{\text {star }}>6.5$ results in the detection of all objects except UMa I and no false positives; in the latter case, setting $S_{\text {star }}>6.0$ includes all objects except CVn II, Leo IV, LeoT, UMa I and no false positives.

Boo II, found by Walsh et al. (2007), is problematic for our algorithm. Boo II contains a very sparsely populated giant branch, and so the brightest stars are sub-giants and turn-off stars at colors of $g-r<0.5$. Given our preferred cuts, Boo II is undetected. It can nonetheless be found with our algorithm, but only by optimizing the color and magnitude cuts, for example, to $g-r<0.5$ and $21<r<23$.

\section{APPLICATION TO SIMULATED DATA}

To test our detection algorithm, we carry out an extensive set of simulations in which we add mock dwarf galaxy satellites and globular clusters to the SDSS DR5 catalog. In particular, we add to the catalog the $g$ and $r$ magnitudes of stars from the simulated objects, at specified right ascensions and declinations. These augmented catalogs are then fed through our automated pipeline, and the number of stellar overdensities with significance above the threshold is calculated as a function of distance, size and luminosity. We explore how changes in the $g-r$ color cuts and kernel sizes ( $\sigma_{1}$ from Eq. 7) affect the efficiency of the algorithm.

The $g$ and $r$ photometry of all simulated objects is based on that of the globular cluster M92. The left panel of Figure 8 shows the color-magnitude diagram (CMD) of M92, together with a main-sequence and red giant branch ridgeline from Clem (2006), to which we have added a horizontal branch ridgeline. From the $r$-band data, we construct a main-sequence and red giant branch luminosity function and approximate it with a smooth fit, as shown in the middle panel of Figure 8. We also determine the luminosity function for the stars on the horizontal branch ridgeline. We populate the ridgelines using the luminosity function. The choice is appropriate, as M92 (12 Gyrs, $[\mathrm{Fe} / \mathrm{H}] \approx-2)$ is typical of the old, metal-poor populations in the Milky Way satellites (see e.g. van den Bergh 2000). Additionally, we add a scatter in $r$ - and $g$ - magnitudes, derived from a fit to the errors in the SDSS point-spread function photometry, as illustrated in the right panel of Figure 8 ,

The spatial distribution of stars in the simulated objects is chosen to follow a Plummer law, which is a reasonable fit to most of the Milky Way dwarf spheroidals (see e.g. Irwin \& Hatzidimitriou 1995; Klevna et al. 2002). For ellipticities less than 0.5 - which corresponds to the most flattened of the SDSS discoveries, Hercules and Ursa Major II (Zucker et al. 2006b; Belokurov et al. 2007) - the detection efficiency of objects barely changes with ellipticity. The Plummer radius, luminosity and distance are chosen to cover uniformly in logarithmic space the following ranges: Plummer radius $1 \mathrm{pc}<r_{h}<$ $1 \mathrm{kpc}$, luminosity $-11 \lesssim M_{v} \lesssim 0$ and heliocentric distance $10 \mathrm{kpc}<D<1$ Mpc. We generate 8000 galaxies with random right ascension and declination within the DR5 footprint. We then split the simulated sample into 20 distance bins to eliminate overlap between simulated objects. The stars from the simulated galaxies are added to the DR5 stellar catalog. Figure 9 shows mock CMDs for simulated objects matching the recently discovered dwarf galaxies Canes Venatici I, Hercules and Ursa Major II (Zucker et al. 2006a; Belokurov et al. 2007; Zucker et al. 2006b). These are good approximations to the observed CMDs of these objects.

In our simulations, we test several inner kernel sizes. The reason is that for a given distance, the kernel size gives rise to an optimum physical size of the detectable objects. For example, at a distance of $50 \mathrm{kpc}$, a kernel size of $4^{\prime}$ corresponds to a physical size of $\simeq 60 \mathrm{pc}$. As we want our algorithm to be sensitive to objects of different 

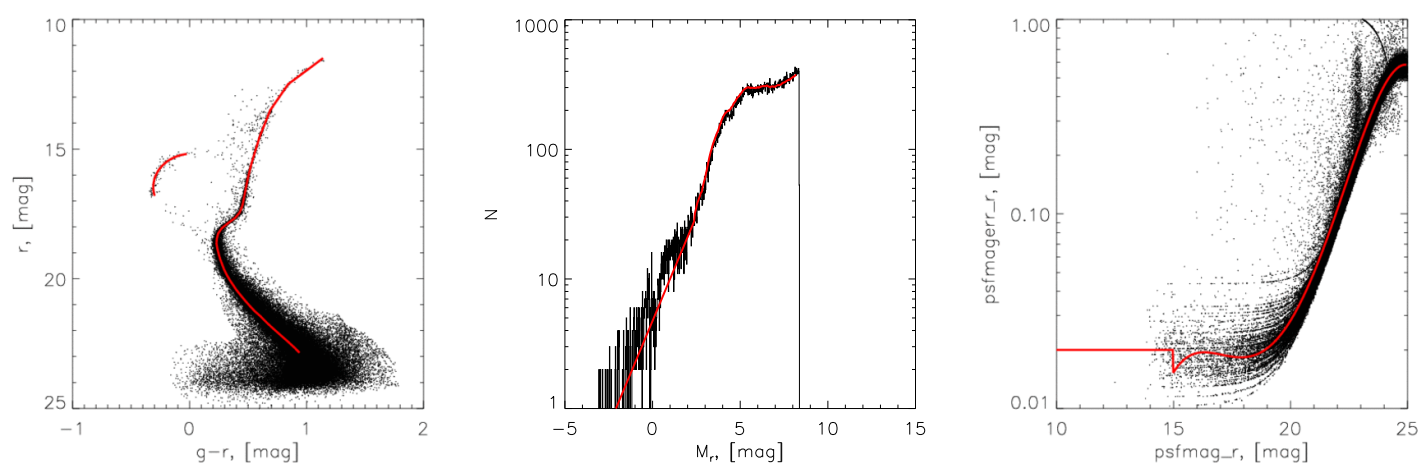

FIG. 8.- Left: M92 color-magnitude data from Clem (2006) used as a template for our simulated Milky Way satellites, together with the ridge line for the main sequence and red giant branch. The ridge line for the horizontal branch is our fit to Clem's (2006) data. Center: The observed luminosity function of main-sequence and red giant branch stars in M92, together with our model fit of the luminosity function used in the simulations. Right: The photometric errors of the SDSS $r$-band photometry and our model fit used in the simulations
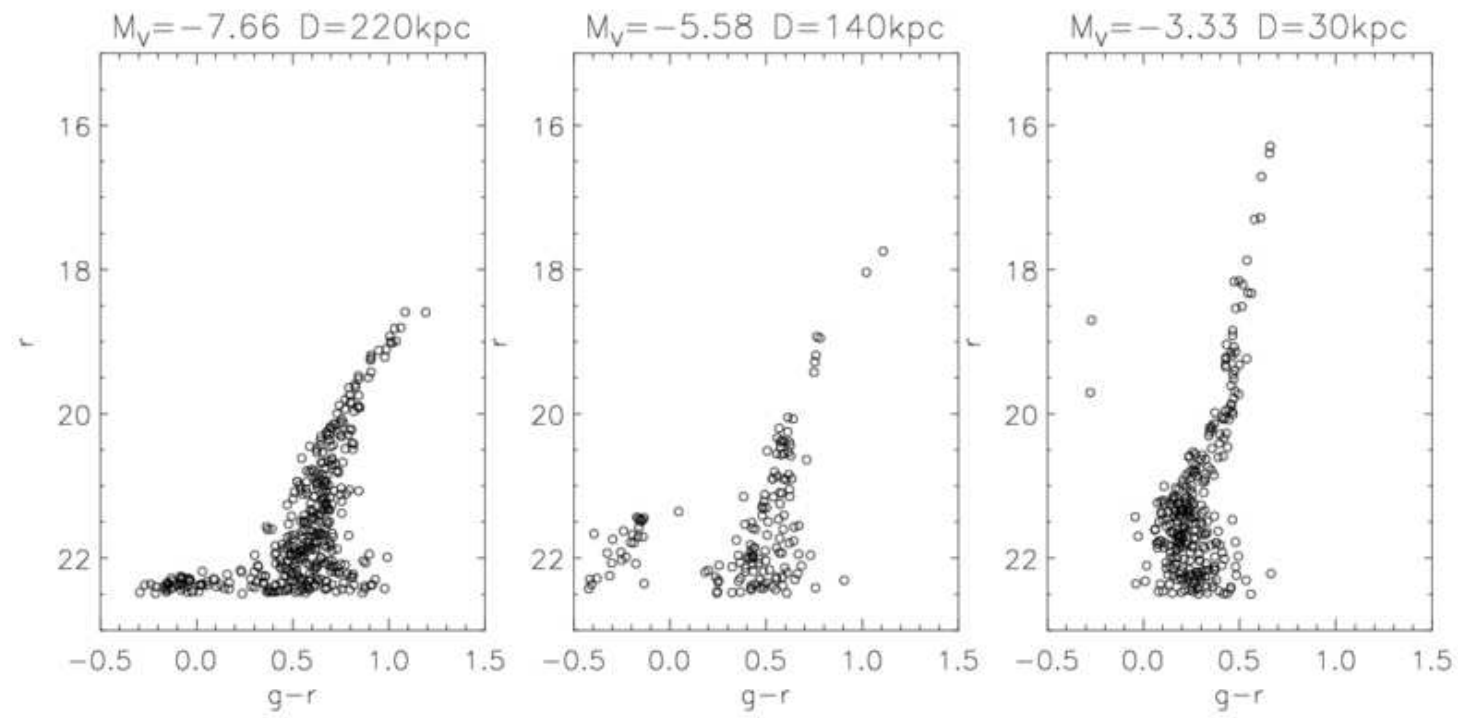

FIG. 9.- Simulated color-magnitude diagrams for hypothetical Milky Way satellite galaxies with properties close to those of Canes Venatici I, Hercules and Ursa Major II-the actual color-magnitude diagrams of these galaxies are given in Zucker et al. (2006a) b) and Belokurov et al. (2007).

sizes, we use three different inner kernel sizes, namely $\sigma_{1}=2^{\prime}, 4^{\prime}$ and $8^{\prime}$. An object is considered to be detected if it is above a threshold on the map convolved with at least one of the kernels (the threshold for the $2^{\prime}$ kernel is 6.50, for the $4^{\prime}$ kernel -5.95 and for the $8^{\prime}$ kernel 6.00 , see Section 3). We refer to this procedure as the combined kernel. This is equivalent to the algorithm used in the previous Section, because the list of detections for $2^{\prime}, 4^{\prime}$ and $8^{\prime}$ kernels includes all the known dwarfs.

Figure [10 shows two-dimensional efficiency maps as a function of luminosity and size in seven distance bins spanning the range $8 \mathrm{kpc}$ to $1 \mathrm{Mpc}$. For Figure 10, we have used the color cut of $g-r<1.2$ and the combined kernel, together with an outer kernel of size $\sigma_{2}=60^{\prime}$. Black corresponds to zero detection efficiency, and white to unit efficiency. The locations of the known Milky Way globular clusters and dwarf galaxies in this parameter space are recorded as red triangles and circles. While a number of known objects lie well within the efficiency boundary, some of the recent discoveries lie close the boundary. It is evident that there is no steady gradi- ent in efficiency, but rather a steep boundary between detectability and non-detectability. In fact, the primary contribution to the finite-extent of the gradient visible in the Figures is produced by the significant extent of the individual distance bins (the width of the distance bins is 0.3 dex). The pixel size in magnitude is 0.8 , and in $\log r_{\mathrm{h}}$, it is 0.3 . This means that there are typically 10 objects in each bin and so we expect moderate fluctuations due to shot noise.

As the efficiency changes so quickly near the boundary, and as several objects lie close to this zone, we carried out more detailed simulations on objects similar to the known dwarfs. We created 1000 Monte Carlo realizations of each of the known dwarfs, and fed them into the pipeline. Table 2 lists the derived detection efficiencies for each object. The detection efficiencies are $\gtrsim 50$ $\%$, with the sole exception of Boo II, confirming our assertion that the known satellites possess high detection probabilities.

For the regime in which objects are larger than the kernel size, some of the stars belonging to the satellite 


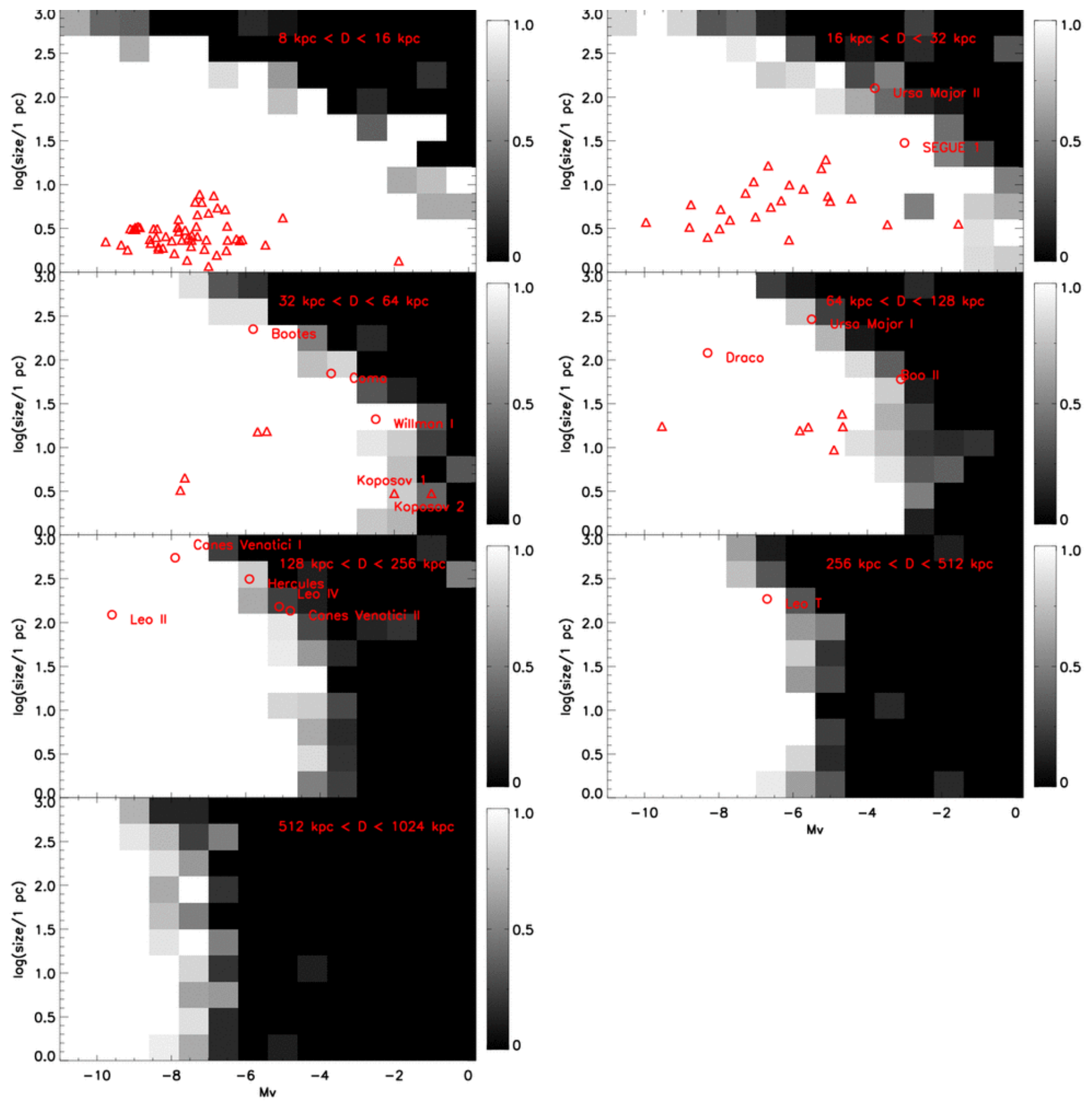

FIG. 10.- Detection efficiency maps for Milky Way satellites, shown as a function of luminosity and size for different distance bins. White indicates 100\% detection efficiency, black indicates $0 \%$. Red circles mark the locations of the known dwarf galaxies, red triangles the known globular clusters (data taken from Harris (1996)). Notice that many of the very recent SDSS satellite galaxy discoveries occur near the boundary, where the detection efficiency is changing rapidly. 
TABLE 1

Objects Detected and Their Significances

\begin{tabular}{|c|c|c|c|c|}
\hline Right ascension & Declination & $S_{\text {star }}$ & $S_{\text {gal }}$ & Name \\
\hline 205.539 & 28.382 & 170.13 & 8.24 & NGC 5272 \\
\hline 168.355 & 22.148 & 170.08 & 19.16 & Leo II \\
\hline 198.220 & 18.159 & 165.00 & 16.48 & NGC5024 \\
\hline 152.100 & 12.289 & 123.86 & 14.94 & Leo I \\
\hline 199.104 & 17.696 & 122.09 & 5.59 & NGC 5053 \\
\hline 211.359 & 28.527 & 121.91 & 5.61 & NGC 5466 \\
\hline 229.006 & -0.130 & 115.10 & 19.48 & Pal5 \\
\hline 260.038 & 57.914 & 100.02 & 15.64 & Draco \\
\hline 250.426 & 36.467 & 94.10 & 2.80 & NGC 6205 \\
\hline 322.483 & 12.147 & 87.58 & 13.30 & NGC7078 \\
\hline 182.516 & 18.544 & 79.40 & 7.58 & NGC 4147 \\
\hline 260.008 & 57.765 & 75.07 & 11.27 & Draco \\
\hline 114.534 & 38.873 & 72.28 & 6.80 & NGC 2419 \\
\hline 323.212 & -0.865 & 65.52 & -1.75 & NGC 7089 \\
\hline 187.670 & 12.395 & 59.69 & 3.25 & NGC 4486 \\
\hline 202.011 & 33.549 & 44.12 & 1.68 & CVn I \\
\hline 187.419 & 8.003 & 37.18 & 0.93 & NGC 4472 \\
\hline 149.834 & 30.742 & 28.13 & 12.65 & Leo A \\
\hline 190.698 & 2.682 & 27.73 & 5.51 & NGC 4636 \\
\hline 114.608 & 21.581 & 25.88 & -6.25 & NGC 2420 \\
\hline 259.027 & 43.063 & 22.76 & -5.60 & NGC 6341 \\
\hline 183.904 & 36.310 & 20.43 & 7.49 & NGC 4214 \\
\hline 185.036 & 29.286 & 17.28 & 0.63 & NGC 4278 \\
\hline 210.010 & 14.503 & 16.95 & 0.32 & Boo I \\
\hline 190.773 & 11.598 & 16.84 & -1.52 & NGC $4647 / 4637 / 463$ \\
\hline 186.368 & 12.909 & 14.28 & 0.76 & NGC 4374 \\
\hline 178.814 & 23.371 & 13.90 & 19.34 & Abell 1413 \\
\hline 186.444 & 33.539 & 13.10 & 16.06 & NGC 4395 \\
\hline 148.904 & 69.081 & 13.08 & 19.85 & NGC 3031 \\
\hline 162.325 & 51.051 & 13.07 & 0.11 & Willman 1 \\
\hline 242.741 & 14.956 & 12.52 & -0.07 & Pal 14 \\
\hline 186.315 & 18.181 & 11.59 & -3.72 & NGC 4382 \\
\hline 132.830 & 63.124 & 11.40 & 0.92 & UMa II \\
\hline 186.745 & 23.913 & 11.22 & -1.02 & Coma Berenices \\
\hline 143.721 & 17.058 & 10.96 & 3.85 & Leo $\mathrm{T}$ \\
\hline 188.911 & 12.544 & 10.91 & -0.97 & NGC 4552 \\
\hline 210.691 & 54.332 & 10.67 & 13.53 & NGC 5457 \\
\hline 151.369 & 0.070 & 10.64 & 4.11 & Pal 3 \\
\hline 186.109 & 7.294 & 9.85 & -0.76 & NGC 4365 \\
\hline 172.319 & 28.961 & 9.53 & 0.26 & Pal 4 \\
\hline 247.764 & 12.789 & 8.91 & 1.05 & Hercules \\
\hline 197.870 & -1.335 & 8.23 & 22.00 & Abell 1689 \\
\hline 194.292 & 34.298 & 7.39 & -4.61 & CVn II \\
\hline 196.743 & 46.569 & 7.30 & 8.29 & Abell 1682 \\
\hline 193.379 & 46.415 & 6.71 & -2.41 & Candidate X \\
\hline 168.146 & 43.440 & 6.52 & -3.22 & Candidate $\mathrm{Y}$ \\
\hline 352.182 & 14.714 & 6.39 & 1.47 & Pegasus \\
\hline 202.388 & 58.404 & 6.37 & -0.59 & NGC 5204 \\
\hline 225.323 & 1.672 & 6.34 & 1.36 & NGC 5813 \\
\hline 187.038 & 44.090 & 6.13 & -2.49 & NGC 4449 \\
\hline 173.235 & -0.554 & 6.10 & 2.92 & Leo IV \\
\hline 0.807 & 16.097 & 6.09 & -2.66 & NGC 7814 \\
\hline 179.144 & 21.049 & 6.05 & 1.14 & Candidate Z \\
\hline 184.843 & 5.786 & 6.04 & -1.25 & NGC 4261 \\
\hline 149.993 & 5.316 & 6.03 & 2.12 & Sextans B \\
\hline 139.470 & 51.718 & 5.97 & 17.86 & Abell 773 \\
\hline 179.223 & 23.379 & 5.96 & 5.22 & galaxy cluster \\
\hline 158.695 & 51.918 & 5.95 & 3.72 & UMa I \\
\hline
\end{tabular}

are missed by the window function, and for such objects the detectability is determined by the number of stars within the window function, i.e. the surface brightness. This effect produces the surface brightness limit seen in Figure 10. For objects smaller than the kernel size, all the stars are within the window function regardless of the size of the objects, therefore for such objects, the detectability doesn't depend on physical size, but depends only on the total number of stars, i.e. the luminosity. This effect produces the rapid change in detection efficiency at fixed absolute magnitude evident in Figure 10. These two regimes can be modeled with thresholds in

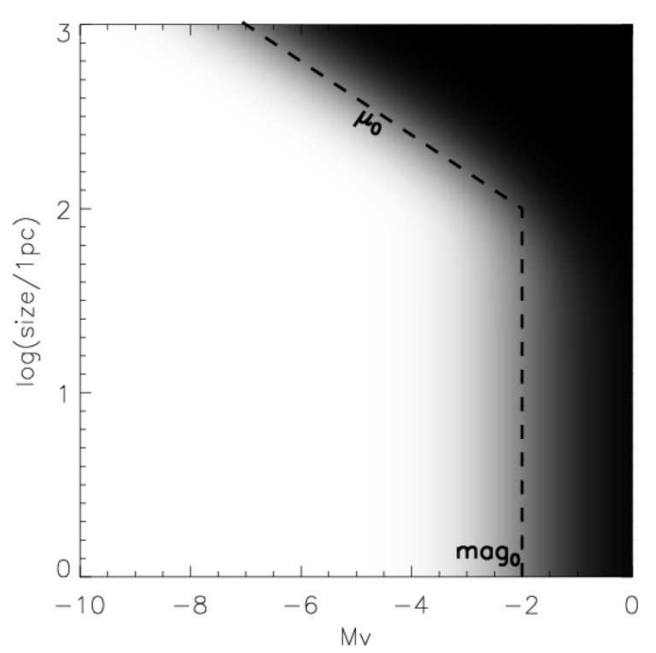

FIG. 11.- Characterizing the satellite galaxy detectability: this illustrative figure shows the model function $\epsilon\left(M_{V}, \mu\right)$ (from Eq. 8) used to fit the observed detection efficiencies from the simulations and demonstrating the role played by the thresholds $M_{V, \lim }$ and $\mu_{\mathrm{lim}}$. The function parameters used to produce the plot 38were $M_{V, \text { lim }}=-2 \mathrm{mag}, \mu_{\mathrm{lim}}=29.5 \mathrm{mag} \operatorname{arcsec}^{-2}, \sigma_{M}=1 \mathrm{mag}$, $\sigma_{\mu}=1 \mathrm{mag}$

surface brightness and absolute magnitude by adopting a functional form:

$$
\epsilon\left(M_{v}, \mu\right)=G\left(\frac{M_{V}-M_{V, \lim }}{\sigma_{M}}\right) G\left(\frac{\mu-\mu_{\lim }}{\sigma_{\mu}}\right),
$$

where $G$ denotes the Gaussian integral, which is defined as

$$
G(x)=\frac{1}{\sqrt{2 \pi}} \int_{x}^{\infty} \exp \left(-t^{2} / 2\right) d t .=\frac{1}{2} \operatorname{erfc}\left(\frac{x}{\sqrt{2}}\right)
$$

To describe the detectability in each distance bin, there are four parameters that are fitted - namely the detection thresholds in surface brightness $\mu_{\text {lim }}$ and absolute magnitude $M_{V \text {,lim }}$, together with their widths $\sigma_{\mu}$ and $\sigma_{M}$. As an illustrative example, the grey-scale map of the efficiency function $\epsilon\left(M_{V}, \mu\right)$ from Eq. 8 is shown in Figure 11, with dashed lines indicating the thresholds. Note the shape of the detection boundary, with the prominent "knee", which corresponds to the boundary between the two detection regimes for objects of different sizes at fixed distance, as described above.

The two key parameters for the detection pipeline are the inner kernel size $\sigma_{1}$ and the color cut applied to the source catalogs. The top two panels of Figure 12 show the dependence of $M_{V \text {, lim }}$ and $\mu_{\lim }$ on distance, when convolved with the three different inner kernels. For a given kernel, the limiting magnitude declines roughly linearly with the logarithm of distance. Objects at the limiting magnitude have an apparent size that is smaller than the kernel size and their detection significance is reduced by the background contribution. Shrinking the kernel size removes some of the background and increases the significance of fainter satellites. The dependence of the $M_{V, \text { lim }}$ and $\mu_{\text {lim }}$ on distance for the combined kernel is not plotted, because in the top left panel of Figure 12 the combined kernel basically follow the dependence of $2^{\prime}$ kernel and in the top right panel of Figure 12 the combined kernel follow the dependence of $8^{\prime}$ kernel. This is illustrated 

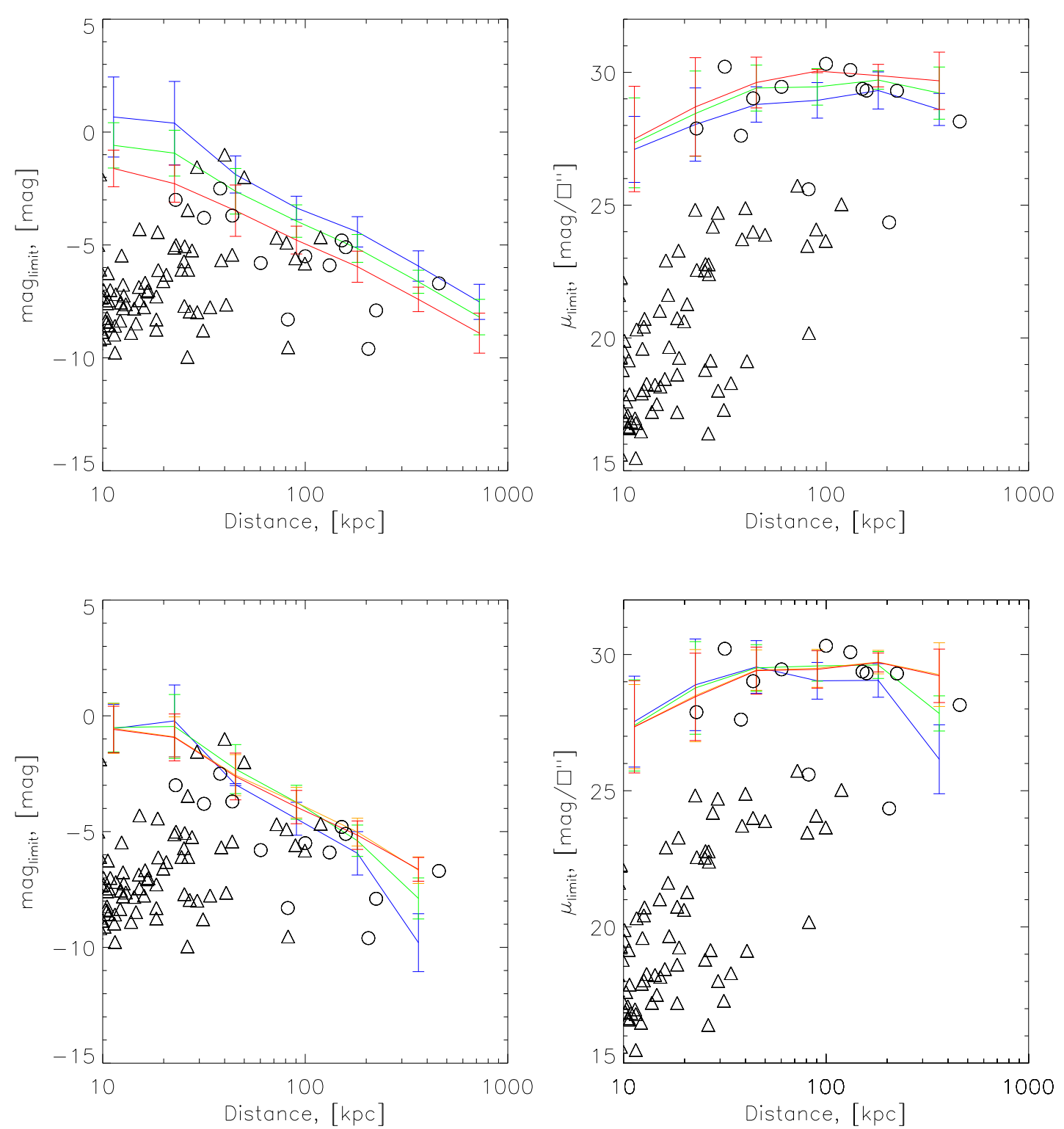

FIG. 12. - The known satellites and globular clusters shown in two-dimensional plots of Galactocentric distance versus absolute magnitude (left) and surface brightness (right). Circles mark the locations of the known dwarf galaxies, triangles the globular clusters. The error bars show either $\sigma_{M}$ or $\sigma_{\mu}$ derived from our model fits (see Eq. 8). The detectability of the objects depends on their location relative to the limiting absolute magnitude (left) and surface brightness (right) as a function of Galactocentric distance for each kernel size/color cut employed in the search. Upper panels: The three lines show the detection limits for different sizes of the inner Gaussian in the kernel (blue $-2^{\prime}$, green $-4^{\prime}$, red $\left.-8^{\prime}\right)$. Lower panels: The four lines show the detection limits for the different $g-r$ color cuts employed (black -0.2 , blue -0.4 , green -0.6 , orange -0.9 , red -1.2 ) and fixed kernel size of $4^{\prime}$.

in the top left panel of Figure [12, where it is clear that a smaller kernel allows us to detect fainter objects. However, as the top right panel shows, this is at the expense of satellite size. Larger kernels pick up more stars from extended objects and hence reach fainter surface brightness. When combining different kernels in the pipeline, the overall limits in surface brightness and absolute magnitude (2', 4', 8', see Section 3) can be approximated by the blue line in the top left panel of Figure 12 and the red line in the top right panel of Figure 12. Smaller kernels allow the detection of galaxies that are low in absolute magnitude, and larger kernels allow the detection of galaxies that are fainter in surface brightness. It is also reassuring to see that the error bars $\sigma_{M}$ are of the same order as the difference in the limiting magnitude moving to a neighboring bin.

We explore the effects of changing color cuts and report the results in the bottom two panels of Figure 12. The color cut of $g-r<0.4$ can improve slightly the magnitude limit for nearby objects by selecting turn-off stars. This improvement deteriorates rapidly as we exhaust the supply of turn-off stars. At larger distances, red color cuts like $g-r<1.2$ are more efficient at picking up giant stars. The same effect explains the drop in $\mu_{\lim }$ and $M_{V \text {,lim }}$ at large distances for bluish color cuts. Our choice of color cut $g-r<1.2$ is conservative, mostly eliminating thin disk stars, and is, overall, the best-behaved and most robust. It also allows us to minimize the influence of metallicity and age changes in the stellar population of the satellites. 
TABLE 2

DeteCtion EFFiciencies of

Simulated ObJects

RESEMBLING KNOWN

SATELlites.

\begin{tabular}{cc}
\hline \hline Object & Efficiency \\
\hline Bootes & 1.00 \\
Draco & 1.00 \\
Leo I & 1.00 \\
Leo II & 1.00 \\
SEGUE 1 & 1.00 \\
Canes Venatici I & 0.99 \\
Willman I & 0.99 \\
Coma & 0.97 \\
Koposov 1 & 0.90 \\
Leo IV & 0.79 \\
Ursa Major II & 0.78 \\
Leo T & 0.76 \\
Hercules & 0.72 \\
Ursa Major I & 0.56 \\
Koposov 2 & 0.48 \\
Canes Venatici II & 0.47 \\
Boo II & 0.20 \\
& \\
\hline
\end{tabular}

TABLE 3

Limiting Satellite Absolute Magnitude ANd Surface BRIGHTNESS AS A FUNCTION OF DisTANCE

\begin{tabular}{ccc}
\hline \hline $\begin{array}{c}\text { Distance } \\
\mathrm{kpc}\end{array}$ & $M_{V, \text { lim }}$ & $\begin{array}{c}\mu_{\text {lim }} \\
\mathrm{mag} / \square^{\prime \prime}\end{array}$ \\
\hline 11 & 0.6 & 27.5 \\
22 & 0.4 & 28.7 \\
45 & -1.9 & 29.6 \\
90 & -3.4 & 30.0 \\
180 & -4.4 & 29.9 \\
260 & -5.9 & 29.9 \\
720 & -7.5 & 29.6 \\
\hline
\end{tabular}

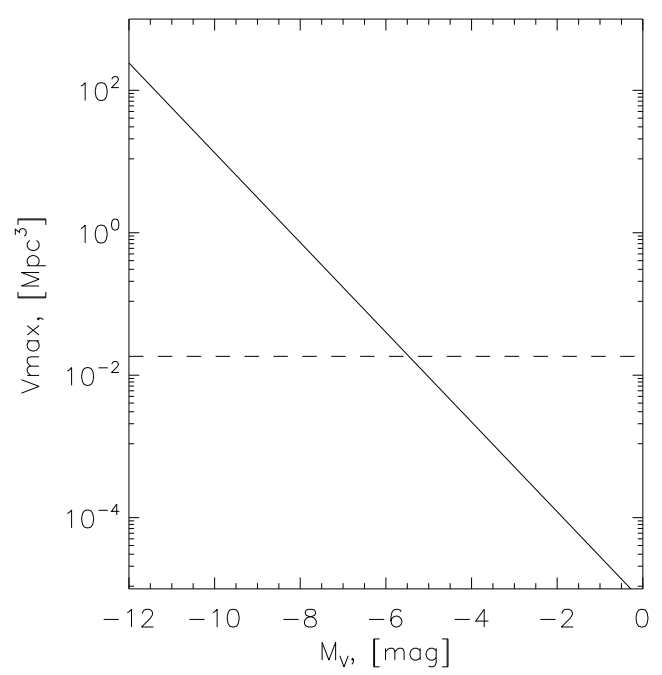

FIG. 13.- The accessible volume within the DR5 footprint for galaxies with different luminosities and surface brightnesses $\mu_{\mathrm{lim}}$ $\mu \lesssim 30 \mathrm{mag} / \square^{\prime \prime}$ (see Figure 12 The volume limited by the virial radius $(280 \mathrm{kpc})$ and within DR5 is shown by the dashed line.

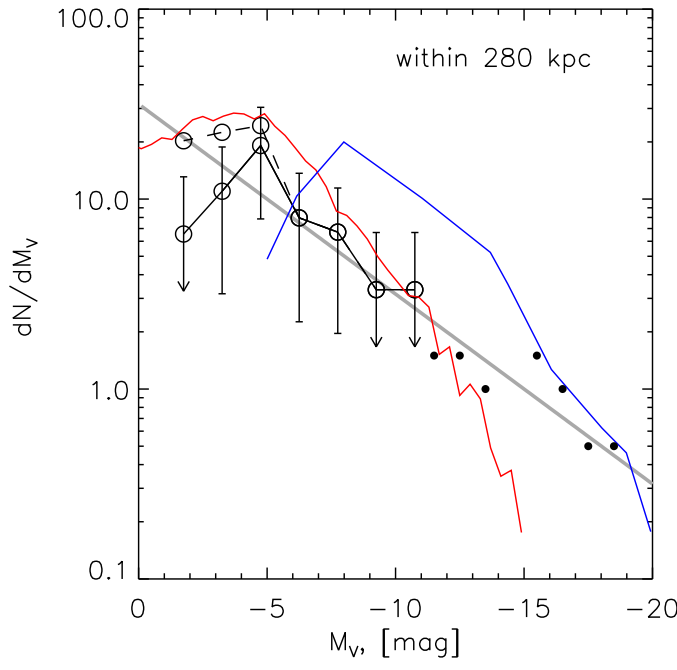

FIG. 14. - The luminosity functions of Milky Way satellite galaxies within $\sim 280 \mathrm{kpc}$ (virial radius) inferred from our analysis under the assumption of two different radial distributions of satellites, NFW-like (solid black line) and isothermal (dashed black line). The calculation uses the satellite list and the volume correction factor obtained with the pipeline using the cuts $r<22.5$ and $S_{\text {star }}>5.95$. The arrows on error bars indicate that there is only one galaxy in the particular bin, and so the Poisson error is formally $100 \%$. The theoretical prediction of Figure 1 of Benson et al. (2002) is shown in a red line, and the prediction of Somerville (2002) for $z_{\text {reion }}=10$ is shown as a blue line. Additionally, the luminosity function for the bright $\left(M_{V}<-11\right)$ satellites of the Milky Way sampled over the whole sky together with the bright M31 satellites within $280 \mathrm{kpc}$ from Metz et al. (2007) is plotted with filled small symbols (the list of plotted objects consists of Sgr, LMC, SMC, Scu, For, LeoII, LeoI, M32, NGC 205, And I, NGC 147, And II, NGC 185, And VII, IC 10). The function $d N / d M_{V}=10 \times 10^{0.1\left(M_{V}+5\right)}$ is shown in grey.

\section{THE LUMINOSITY FUNCTION}

\subsection{Analysis of the detection efficiency maps}

With an understanding of which satellite galaxies can be detected in SDSS DR5, together with our sample of actual detections, we can now estimate the luminosity function of faint Milky Way satellites. We start by re-examining the efficiency maps in Figure 10, where the locations of the known Milky Way globular clusters and dwarf galaxies are overplotted as red triangles and circles respectively. We can conclude that within the DR5 footprint there are certainly no bright satellites (either globulars or galaxies) nearby $(D<32 \mathrm{kpc})$ that have eluded discovery. However, the disrupting galaxy UMa II (Zucker et al. 2006b) provides a clue as to the likely locations of remnants. It is still possible that disrupted galaxies remain undiscovered nearby. They can lurk in the black portions of the uppermost two panels of Figure 10,

All that has survived in the inner Galaxy $(8<D<16$ $\mathrm{kpc}$ ) is a population of globular clusters, which occupy a small region in the luminosity and size parameter space. They are predominantly old globular clusters belonging to the bulge. Only the densest survive against the disruptive effects of Galactic tides and shocking, which is illustrated by the apparent size bias. Notice that the datapoints lie well away from the detection boundary, suggesting that the sample is complete at least within $8<D<16$. Moving outwards 
$(16<D<32 \mathrm{kpc})$, the globular clusters belong to the halo and may have been accreted (Mackev \& Gilmore 2004). Their size distribution is broader. Some of these objects are in the process of disruption, such as Pal 5 and NGC 5466 (Odenkirchen et al. 2001; Dehnen et al. 2004; Belokurov et al. 2006c; Fellhauer et al. 2007). The very faint and distant globular clusters discovered recently by Koposov et al. (2007) are visible in the third panel of Figure $10(32<D<64 \mathrm{kpc})$ right on the border of detectability. Further such sparse globulars may remain undetected.

Beyond $30 \mathrm{kpc}$, the dwarf spheroidals begin to appear. The long-known dwarfs such as Draco and Sculptor lie far from the boundary, in regions of the luminosity and size parameter space where the DR5 search efficiency is unity. However, all the recent SDSS discoveries, such as Canes Venatici I, Bootes and Hercules, lie close to the detection boundary, where the efficiency declines rapidly from unity to zero. Belokurov et al. (2007) claimed that there is a paucity of objects with half-light radii between $\sim 40 \mathrm{pc}$ and $\sim 100 \mathrm{pc}$. Our calculations support the idea that the gap is real and not produced by selection effects. If there were objects with radii between $\sim 40 \mathrm{pc}$ and $\sim 100 \mathrm{pc}$, there is a broad range of parameter space in which they would have been found.

Although most of the new detections lie in the gray areas of the plot, the empty white regions with unit efficiency are telling us something important. There are swathes of the parameter space in which we would have detected objects if they existed. For example, there are very few bright objects $\left(M_{v}<-6\right)$. The absence of detections of bright objects does by itself provide a strong constraint on the luminosity function of Milky Way satellites. There also do not appear to be any analogues of the extended, luminous star clusters found in M31 by Huxor et al. (2005). Although SDSS data may still contain evidence for further, hitherto unknown, dwarf galaxies, it is unlikely that their nature can be unambiguously established without substantial quantities of followup imaging. We emphasize that, since we never probe fainter than a certain surface brightness limit, an even larger population of very low surface brightness galaxies - which can not be detected with SDSS - may exist.

\subsection{Estimation of the Luminosity function}

Figure 13$]$ shows the accessible volume for galaxies of different luminosities probed by our algorithm (which in practice is a function mostly of the luminosity) within the SDSS DR5 footprint. As the logarithm of distance scales roughly linearly with limiting magnitude (see Figure 12), so does the logarithm of the accessible volume. Using this, and the fact that the SDSS survey covers $\sim 1 / 5$ of the sky, we can convert the set of known objects into a volume corrected luminosity function ${ }^{6}$.

The observed luminosity function is constructed using all the well-established dwarf galaxies in DR5, namely Leo II, Draco, Leo I, CVn I, Boo I, Hercules, UMa II,

\footnotetext{
6 The existing data on the globular cluster population indicate that at least some globular clusters have complicated metallicity, age distributions and kinematics and may in fact be stripped nuclei of dwarf galaxies (Zinnecker et al. 1988; Piotto et al. 2007). The selection of such objects which are considered as dwarf galaxies is an additional source of uncertainty in any luminosity function determination.
}

Com, CVn II, Leo T, UMa I, Leo IV as well as the possible dwarf Willman 1 . Segue 1 is not used because it is not in DR5 (Belokurov et al. 2007), and Boo II is not used because it is not detected with our adopted identification-pipeline parameters. All the satellites included in our calculation have a surface brightness of at least $30 \mathrm{mag} \operatorname{arcsec}^{-2}$. To relate the observed number of satellite galaxies in our sample to the total number of satellites in the Milky Way halo, it is necessary to adopt an underlying radial distribution of satellite galaxies (see Appendix). In a given magnitude interval, we know the observed number of satellites within $V_{\max }\left(M_{v}\right)$ from Figure 13, together with their detection efficiencies from Table 2, If we assume a number density law $n(r)$ for the satellites, then its normalization at each magnitude interval can be fixed by integrating the density law out to $V_{\max }$. The total number of satellites within $280 \mathrm{kpc}$ (the virial radius of the halo) is now the integral of the density law out to this limit. Figure 14 shows the results of the calculation for two such density laws. The dashed line shows the luminosity function assuming the satellites are distributed in an isothermal sphere (namely, $n(r) \propto 1 / r^{2}$ ). The solid line shows the luminosity function if the density fall-off is steeper at large radii $\left(n(r) \propto 1 / r^{3}\right.$, like Navarro-Frenk-White profile, although to prevent the $1 / r^{3}$ profile from diverging in the MW center we use $n(r) \propto r^{-2}\left(r+r_{\mathrm{c}}\right)^{-1}$ with the core radius $r_{c}=10 \mathrm{kpc}$ ). Of course, the nature of some of the objects we have included in the dwarf galaxy luminosity function is still uncertain - in particular, Willman 1 may be a globular cluster, although Martin et al. (2007) provide evidence for a metallicity spread and dark matter content. It is unclear whether Leo $\mathrm{T}$ should be included or excluded, as it is most likely a transition object with rather different properties from the other dwarf spheroidal galaxies in our sample. The error bars in Figure 14 are given by the square root of the number of datapoints in the absolute magnitude interval divided by the volume correction factor. At the bright end, the error bars are large, since we have only two objects with $M_{v}<-9$, namely Leo I $\left(M_{v}=-11.5\right)$ and Leo II $\left(M_{v}=-9.6\right)$. At the faint end, the error bars are also large because of the substantial volume correction factor. In Figure 14, we show the luminosity function for satellites within $280 \mathrm{kpc}$ (a proxy for a Milky Way virial radius (Klypin et al. 2002; Benson et al. 2002)). To define the bright end of the luminosity function, which cannot be reliably determined from our data since DR5 does not contain dwarfs brighter than $M_{V} \sim-11$, we have also included in Figure 14 the estimate of the luminosity function (filled points) for the bright satellites of the Milky Way sampled over the full sky, together with the bright M31 satellites within $280 \mathrm{kpc}$ from Metz et al. (2007). In Figure 14, we also overplot the power-law function $d N / d M_{V}=10 \times 10^{0.1\left(M_{V}+5\right)}$, which approximates the datapoints in the range of $-19<M_{V}<-2$ (with probably some flattening at $\left.M_{V} \sim-4\right)$. The integration of this power-law gives approximately 45 dwarfs brighter than -5.0, and 85 dwarfs brighter than -2.0.

There are a number of theoretical predictions of the luminosity function of the Local Group in the literature. For example, Somerville (2002) shows the results of semi-analytic galaxy formation calculations, includ- 
ing the effects of supernova feedback and photoionization. The luminosity function from Somerville (2002) for $z_{\text {reion }}=10$ (Page et al. 2007) are plotted with blue line in Figure 14. Although the numbers of luminous satellites are in reasonable agreement with the data, the shape of the luminosity function is not. All Somerville's (2002) luminosity functions turn over at $M_{v} \approx-9$ or brighter, depending on the epoch of reionization, whereas the luminosity function derived in Figure 14 turns over fainter than $M_{V} \approx-5$, if at all. Therefore, Somerville's (2002) theoretical calculations overproduce Draco-like objects $\left(M_{V} \approx-10\right)$ by a factor of a few, and underproduce much fainter galaxies like Boo $\left(M_{V} \approx-6\right)$ by almost an order of magnitude.

Benson et al. (2002) also provides calculations of the luminosity function of the Milky Way satellites, including the effects of tidal disruption as well as photoionization. They report the luminosity functions for dwarfs with a range of of different central surface brightness cuts, namely $18,20,22,24$ and 26 mag $\operatorname{arcsec}^{-2}$, the last of which is plotted in Figure 14 in a red line. At first glance, the fit seems plausible, especially given the size of the error bars on the datapoints. The turn over in Benson et al.'s luminosity function is at $M_{V} \approx-3$ and the numbers of predicted satellites at faint magnitudes are also consistent given the uncertainties. However, Benson et al.'s model significantly underproduces the number of bright satellites. Additionally, Benson et al.'s satellites have a much higher central surface brightness - our SDSS survey corresponds to a surface brightness cut of $\sim 30$ mag $\operatorname{arcsec}^{-2}$. Figure 2 of Benson et al. (2002) does show the luminosity function for all objects, irrespective of surface brightness. Although there has been a large change in the luminosity function on moving from a detection threshold of 22 to $26 \mathrm{mag} \operatorname{arcsec}^{-2}$, there is only a small change on moving from 26 to $\infty$ mag $\operatorname{arcsec}^{-2}$.

\section{CONCLUSIONS}

There have been persistent discrepancies between the observed numbers of Milky Way satellites and the predictions from numerical simulations of galaxy formation for a number of years. Although here has been a cavalcade of discoveries of new Milky Way satellites using the SDSS over the last two years, a systematic search - with quantifiable detection limits and efficiencies - not been undertaken. In this paper we have presented a quantitative search methodology for Milky Way satellite galaxies in SDSS data and have used this method to compute detection efficiency maps, which ultimately allow the construction of the satellite galaxy luminosity function.

In our method, the star count map is convolved with a family of kernels which are the difference of two Gaussians. Intuitively, this algorithm can be understood as constructing an estimate of the local stellar density minus the background. By attaching a statistical significance to the overdensities in the convolved image, this enables us to construct a ranked list of candidates. Although this idea is simple enough, its practical application is hampered by the fact that the separation between stars and galaxies by the SDSS pipeline becomes unreliable at magnitudes fainter than $r \simeq 22.5$. The resulting false positives must be removed by cross-correlating with galaxy catalogs. The significance threshold of peaks in our survey is set by requiring the detection pipeline to produce a "clean" list of Milky Way satellites.

To compute the detection efficiency, we create mock SDSS catalogs with stars from simulated dwarf galaxies and use Monte Carlo methods to estimate recovery as a function of satellite galaxy parameters and heliocentric distance. There is a sharp boundary between detectability and non-detectability. The efficiency maps make clear that there are large domains in parameter space in which objects would have been detected had they existed. In particular, even at heliocentric distances as great as $1 \mathrm{Mpc}$, objects brighter than $M_{v} \sim-8$ would have been detectable in SDSS. Similarly, populations of extended, luminous star clusters would have been found in SDSS, if they existed in the Milky Way.

With the efficiency in hand, we can - for the first time - correct the observed luminosity function of the Milky Way satellites for selection effects and compute the true luminosity function. The number density of satellite galaxies continues to rise well below $M_{V} \sim-8^{m}$; depending on the radial distribution model assumed it may or may not flatten or turn over at $M_{V} \gtrsim-5$. Overall, the luminosity function of all Milky Way satellites may be reasonably well described by a power-law, $d N / d M_{V}=10 \times 10^{0.1\left(M_{V}+5\right)}$ from $M_{V}=-2$ to -18 . This power-law predicts $\sim 45$ satellites brighter than $M_{V}=-5$, and $\sim 85$ satellites brighter than $M_{V}=-2$. The normalization of the luminosity function is in reasonable agreement with the predictions of semi-analytic modeling of galaxy formation, but the shape is not. There also remains a discrepancy in the distribution of surface brightnesses of such objects, in the sense that the semi-analytic models underproduce dwarfs with a central surface brightness fainter than $26 \mathrm{mag} \operatorname{arcsec}^{-2}$.

S. Koposov is supported by the DFG through SFB 439 and by a EARA-EST Marie Curie Visiting fellowship. VB acknowledges the award of a a Postdoctoral Research Fellowship from the Science and Technology Facilities Council (STFC). NWE, PCH and DZ acknowledge support from the STFC-funded Galaxy Formation and Evolution programme at the Institute of Astronomy. We thank Andrew Benson for supplying us with numerical data on his semi-analytic calculations. Funding for the SDSS and SDSS-II has been provided by the Alfred P. Sloan Foundation, the Participating Institutions, the National Science Foundation, the U.S. Department of Energy, the National Aeronautics and Space Administration, the Japanese Monbukagakusho, the Max Planck Society, and the Higher Education Funding Council for England. The SDSS Web Site is http://www.sdss.org/.

The SDSS is managed by the Astrophysical Research Consortium for the Participating Institutions. The Participating Institutions are the American Museum of Natural History, Astrophysical Institute Potsdam, University of Basel, Cambridge University, Case Western Reserve University, University of Chicago, Drexel University, Fermilab, the Institute for Advanced Study, the Japan Participation Group, Johns Hopkins University, the Joint Institute for Nuclear Astrophysics, the Kavli Institute for Particle Astrophysics and Cosmology, the Korean Scientist Group, the Chinese Academy of Sciences (LAMOST), Los Alamos National Laboratory, the Max-Planck-Institute for Astronomy (MPIA), the Max- 
Planck-Institute for Astrophysics (MPA), New Mexico State University, Ohio State University, University of Pittsburgh, University of Portsmouth, Princeton University, the United States Naval Observatory, and the Uni- versity of Washington. This research has made use of the SAI Catalogue Access Services, Sternberg Astronomical Institute, Moscow, Russia

\section{APPENDIX}

\section{THE CALCULATION OF THE CORRECTION TO THE LUMINOSITY FUNCTION}

To calculate the luminosity function of Milky Way satellites within $r_{\mathrm{LF}}=280 \mathrm{kpc}$, we select all the satellites within DR5 which are interior to $r_{\mathrm{LF}}$, and construct the histogram of $M_{V}$ of these objects. From the simulations, we know that not all objects are detected with $100 \%$ efficiency and the histogram $h\left(M_{V}\right)$ is weighted with the object detection efficiencies.

$$
h\left(M_{V}\right)=\sum_{i} \frac{1}{\epsilon_{i}} \delta\left(M_{V}, M_{V, i}\right)
$$

where $\epsilon_{i}$ is the detection efficiency of i-th object, $M_{V, i}$ its luminosity, and $\delta\left(M_{V}, M_{V, i}\right)=1$, if $M_{V}$ and $M_{V, i}$ are within one bin of the histogram, and 0 otherwise.

Figure 12, shows how the maximal accessible distance depends on the galaxy luminosity (the $r_{\max }\left(M_{V}\right)$ function). From this function, we can construct the maximal accessible volume within the DR5 footprint (which covers $1 / 5$ of the sky) as a function of galaxy luminosity, namely $V_{\max }\left(M_{V}\right)=4 \pi / 3 f_{D R 5} r_{\max }^{3}\left(M_{V}\right)$ (see Figure 13), where $f_{D R 5}$ is the fraction of the sky covered by DR5. Then we construct the incompleteness correction $c\left(M_{V}\right)$, using the probability distribution of the satellites $n(r)$. When the maximal accessible distance for a galaxy is greater than $r_{\mathrm{LF}}$, the correction is 1, if not it is equal to the ratio of number of satellites within $r_{\max }\left(M_{V}\right)$ to the number of satellites within $r_{\mathrm{LF}}$ :

$$
c\left(M_{V}\right)= \begin{cases}\frac{\int_{0}^{r_{\max }\left(M_{V}\right)} n(r) r^{2} d r}{\int_{0}^{r_{\mathrm{LF}}} n(r) r^{2} d r} & \text { if } r_{\max }\left(M_{V}\right)<r_{\mathrm{LF}} \\ 1 r_{\max }\left(M_{V}\right) \geq r_{\mathrm{LF}}\end{cases}
$$

Finally, the luminosity function is obtained by dividing the histogram of luminosities $h\left(M_{V}\right)$ by the incompleteness correction $c\left(M_{V}\right)$

\section{REFERENCES}

Adelman-McCarthy, J. K., et al. 2006, ApJS, 162, 38

Babaud J., A. P. Witkin, M. Baudin, and R. O. Duda. 1986, IEEE Trans. Pattern Anal. Mach. Intell., 8, 1, 26

Belokurov, V., et al. 2006a, ApJ, 642, L137

Belokurov, V., et al. 2006b, ApJ, 647, L111

Belokurov, V., Evans, N. W., Irwin, M. J., Hewett, P. C., \& Wilkinson, M. I. 2006c, ApJ, 637, L29

Belokurov, V., et al. 2007, ApJ, 654, 897

Benson, A. J., Frenk, C. S., Lacey, C. G., Baugh, C. M., Cole, S. 2002, MNRAS, 333, 177

Bullock J.S., Kravtsov A.V., Weinberg D.H. 2001, ApJ, 548, 33

Clem, J. L. 2006, Ph.D. Thesis,

Dehnen W., Odenkirchen M., Grebel E. K., Rix H.-W. 2004, AJ, 127,2753

Diemand J., Madau P., Moore B. 2005, MNRAS, 364, 367

Fellhauer M., et al. 2007, MNRAS, submitted

Fukugita M., Ichikawa T., Gunn J. E., Doi M., Shimasaku K., Schneider D. P. 1996, AJ, 111, 1748

Koposov, S. E., Glushkova, E. V., \& Zolotukhin, I. Y. 2007, ArXiv e-prints, 709, arXiv:0709.1275, accepted to A\&A

Gunn, J.E. et al. 1998, AJ, 116, 3040

Gunn, J.E. et al. 2006, AJ, 131, 2332

Gnedin, O. Y., Zhao, H., Pringle, J. E., Fall, S. M., Livio, M., \& Meylan, G. 2002, ApJ, 568, L23

Harris W.E., 1996, AJ, 1121487

Huxor, A. P., Tanvir, N. R., Irwin, M. J., Ibata, R., Collett, J. L., Ferguson, A. M. N., Bridges, T., \& Lewis, G. F. 2005, MNRAS, 360,1007

Hogg, D.W., Finkbeiner, D.P., Schlegel, D.J., Gunn, J.E. 2001, AJ, 122,2129

Irwin M. J., Hatzidimitriou D. 1995, MNRAS, 277, 1354

Irwin M. J., et al. 2007, ApJ, 656, L13

Kleyna, J., Wilkinson, M. I., Evans, N. W., Gilmore, G., \& Frayn, C. 2002, MNRAS, 330, 792

Klypin A., Kravtsov A. V., Valenzuela O., Prada F. 1999, ApJ, 522,82
Klypin, A., Zhao, H., \& Somerville, R. S. 2002, ApJ, 573, 597

Koposov, S., \& Bartunov, O. 2006, Astronomical Data Analysis Software and Systems XV, 351, 735

Koposov, S., Bartunov, O., Belinskiy, A., Karpov, S. 2007, Astronomical Data Analysis Software and Systems XVI, 376, 34

Koposov, S., et al. 2007, ApJ, 669, 337

Lindenberg, T., 1993, International Journal of Computer Vision, $11,3,283$

Lindenberg, T., 1998, International Journal of Computer Vision, $30,2,79$

Lupton, R., Gunn, J., \& Szalay, A. 1999, AJ, 118, 1406

Lupton, R., Gunn, J. E., Ivezić, Z., Knapp, G. R., \& Kent, S. 2001, Astronomical Data Analysis Software and Systems X, 238, 269

Mackey, A. D., \& Gilmore, G. F. 2004, MNRAS, 355, 504

Martin, N. F., Ibata, R. A., Chapman, S. C., Irwin, M., \& Lewis, G. F. 2007, MNRAS, 380, 281

Metz, M., Kroupa, P., \& Jerjen, H. 2007, MNRAS, 374, 1125

Moore B., Ghigna S., Governato F., Lake G., Quinn T., Stadel J., Tozzi P. 1999, ApJ, 524, L19

Moore B., Diemand J., Madau P., Zemp M., Stadel J. 2006, MNRAS, 368, 563

Odenkirchen M., et al. 2001, ApJ, 548, L165

Page, L., et al. 2007, ApJS, 170, 335

Pier, J.R., Munn, J.A., Hindsley, R.B, Hennessy, G.S., Kent, S.M., Lupton, R.H., Ivezic, Z. 2003, AJ, 125, 1559

Piotto, G., et al. 2007, ApJ, 661, L53

Schlegel, D. J., Finkbeiner, D. P., \& Davis, M. 1998, ApJ, 500, 525

Silverman, B. W. 1986, Monographs on Statistics and Applied Probability, London: Chapman and Hall, 1986,

Smith, J. A., et al. 2002, AJ, 123, 2121

Somerville, R. S. 2002, ApJ, 572, L23

Stoehr F., White S. D. M., Tormen G., Springel V. 2002, MNRAS, 335, L84

Stoughton, C. et al. 2002, AJ, 123, 485 
Tucker D., et al. 2006, AN, in press

de Vaucouleurs, G., de Vaucouleurs, A., Corwin, H. G., Jr., Buta, R. J., Paturel, G., \& Fouque, P. 1991, Volume 1-3, XII, 2069 pp. 7 figs.. Springer-Verlag Berlin Heidelberg New York,

van den Bergh, S. 2000, The galaxies of the Local Group, by Sidney Van den Bergh. Published by Cambridge, UK: Cambridge University Press, 2000 Cambridge Astrophysics Series Series, vol no: 35, ISBN: 0521651816. ,

Walsh, S. M., Jerjen, H., \& Willman, B. 2007, ApJ, 662, L83

Whiting, A. B., Hau, G. K. T., Irwin, M., \& Verdugo, M. 2007, AJ, 133,715

Willman, B., Dalcanton J., Ivezić Ž., Jackson T., Lupton R., Brinkmann J., Hennessy G., Hindsley R. 2002, AJ, 123, 848
Willman, B., et al. 2005a, ApJ, 626, L85

Willman, B., et al. 2005b, AJ, 129, 2692

York D. G., et al. 2000, AJ, 120, 1579

Zinnecker, H., Keable, C. J., Dunlop, J. S., Cannon, R. D., \& Griffiths, W. K. 1988, The Harlow-Shapley Symposium on Globular Cluster Systems in Galaxies, 126, 603

Zucker D. B., et al. 2006a, ApJ, 643, L103

Zucker D. B., et al. 2006b, ApJ, 650, L41 